INTERNATIONAL DESIGN CONFERENCE - DESIGN 2018

https://doi.org/10.21278/idc.2018.0176

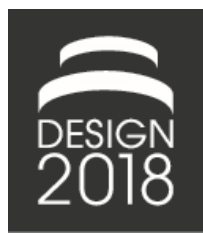

\title{
GEOMETRIC SUBSTITUTE MODELS FOR EFFICIENT SCALING OF DIMENSIONS DURING VEHICLE ARCHITECTURE DESIGN
}

\author{
M. Felgenhauer, C. Angerer, R. Marksteiner, F. Schneider and M. Lienkamp
}

\begin{abstract}
The scaling of component sizes and distances between them, during the early stage of Vehicle Architecture Design, is time- and cost-consuming, due to numerous requirements as well as component and position alternatives. To enable fast scaling of dimensions, this paper introduces the development of empirical and semi-physical geometric substitute models. For over 25 components and distances between them, over 300 vehicles of the A2Mac 1 benchmarking database serve as main data source. The resulting accuracies range from $80 \%$ to $97 \%$ and are applicable for initial architecture investigations.
\end{abstract}

Keywords: design automation, design models, architectural design, early design phase, complex systems

\section{Introduction}

\subsection{Background description}

At the beginning of the development of a vehicle, the Vehicle Concept Development Process takes place. During this early stage, the concept engineers develop a geometric model of the vehicle, from the product idea through to the scaling and positioning of components.

In order to fulfil these tasks, the Vehicle Concept Development Process is divided into three phases: the Vehicle Dimension Design (VDD), the Vehicle Architecture Design (VAD) and the Vehicle Package Design (VPD) (Felgenhauer et al., 2017). The VDD defines the overall dimensions and the passenger positions. Accordingly, this phase determines the available installation space from the outside to the inside. Afterwards, the VAD scales the sizes of the most important components, for example the engine, the gearbox and the cooling system. In addition, the positioning of the components determines the distances between components. The variation of drivetrain concepts and component variants, for example different types of electric machines or combustion engines, lead to multiple architecture alternatives. Within these alternatives, the evaluation of the distances and collisions between components takes place, as well as the alignment with the available installation space. The selection of the best architecture alternative is based on a geometric, functional and economic assessment. Within the selected architecture alternative, the VPD scales and positions the remaining components. Like in the case of Vehicle Architecture Design, concept engineers assess the compliment with the available installation space. Series development and production follow the Vehicle Concept Development Process. 


\subsection{Problem description}

Aside from the definition of the exterior dimensions and the vehicle type during the VDD, the Vehicle Architecture Design influences the new vehicle concept the most. Numerous drivetrain concepts as well as component variants and installation positions create a vast solution space. This variance leads to two interdependent influences, causing high complexity during the VAD:

- High number of requirements: For the vehicle architecture numerous requirements exist, for instance the vehicle acceleration and speed, as well as the crash deceleration.

- High amount of component and position variants: Multiple component and position variants are available during the VAD. For example different engine types, such as electric and combustion engines, as well as engine orientations, e.g. longitudinal and transversal engine installation, are available.

Due to the high number of requirements and variants, the coherences between the requirements and the component sizes, as well as distances between components, are unknown. This is especially true, as the coherences vary for different variants and drivetrain concepts. Consequently, concept engineers struggle to scale component sizes and required distances between components, at the beginning of the VAD. For example, it is difficult to determine the engine length based on vehicle acceleration and speed. Especially, as the engine length depends on the component variant, such as electric and combustion engines and their sub-variants. However, the sizes of the components and the distances between them are essential for the definition of vehicle architectures.

As a starting point of the VAD, concept engineers assume the component sizes and distances between them. For vehicles with similar requirements and component variants, they can base it on previous and competitor vehicles. For new requirements and component variants, the concept engineers determine the dimensions based on assumptions and - if possible - early simulations. Afterwards, the assumed dimensions are iteratively refined. To create and evaluate multiple vehicle architectures, this is done for manifold component variants and drivetrain concepts. Consequently, the definition of the dimensions within the vehicle architecture design is not only difficult but also time- and cost-consuming. Due to limited capacities, it is impossible to holistically consider all available component variants and therefore vehicle architectures.

\subsection{Target}

To enable the fast scaling of dimensions, as a starting point of initial architecture investigations, the target is to develop geometric substitute models for the components and distances between them. Therefore, the authors use empirical data and physical equations to model the coherences between the requirements and the dimensions. Components and distances of combustion, hybrid and electric drivetrain concepts are considered. With the substitute models created, the dimensions are not only continuously scalable based on the requirements but also more precise than early-stage assumptions. Due to the fast prediction of dimensions, a holistic consideration of all component variants is also possible. This enables faster and broader architecture investigations. After the initial phase, the substitute models will be replaced by detailed designs.

\subsection{Research method}

The method presented considers the knowledge and experience of experts from the Vehicle Concept Development Department of AUDI AG and of an interdisciplinary group of researchers focussing on vehicle concepts at the Technical University of Munich.

\section{State of the art}

\subsection{Terminology of vehicle architectures}

In the following, a vehicle architecture defines the body structure as well as dimensions and position of the most important components, such as the engine, gearbox, chassis and cooling system (Fuchs, 2014). In comparison, a topology only defines positions of components (Ried, 2014). 


\subsection{Existing approaches to Vehicle Architecture Design}

The state of the art regarding Vehicle Architecture Design is divided into the generation and analysis of the solution space within one architecture and the generation and comparison of multiple architectures.

Fuchs (2014) is able to generate vehicle architectures for BEV and FCEV vehicles. Using physical coherences and empirical scaling factors he develops substitute models for the electric machine, the hydrogen tank and the high-voltage electrical system. In addition, he can scale the high-voltage battery as well as the fuel cell stack, based on an analytic determination of the required number of modules/stacks as well as reference geometries. For the remaining components, he either uses low-detail component models or existing components. However, the comparison of architectures is not within the scope of his work. Instead he analyses the solution space within one architecture, by variation of component configurations and properties.

Ried (2014) uses a parametric model for the generation of PHEV vehicle architectures. He is able to display three different architectures by the variation of the underbody battery topology. To adapt to different input requirements he uses scalable component models for some components. However, he does not give a detailed overview of the considered components. In addition, he did not publish the data sources, the procedure or level of detail for the modelling of the component models, nor the resulting coherences and their accuracies. The selection within the feasible architectures is based on a cost analysis.

Based on a parametric vehicle model for BEV, Kuchenbuch (2012) uses multi-criteria optimization to generate new architectures. His focus is to identify vehicle architectures with new battery topologies and the best compromise between energy consumption and battery range. A semi-physical battery model calculates the battery capacity and power using reference battery cells. All other component models are based on a database. However, the parametric models' dimensions are limited to 2.5-D.

In 3-D, Matz (2015) uses an optimization algorithm to identify the optimal vehicle architecture for BEV based on the customer requirements and the availability of other public transportation systems. Like Kuchenbuch, he uses a battery model with physical equations and reference cells as well as a component database. In contrary, his focus is on the overall vehicle architecture instead of the battery topology.

\subsection{Research gap}

Several research methods contribute to Vehicle Architecture Design. However, previous authors focus on specific drivetrain concepts and use empirical and physical substitute models for only a few components. In addition, information regarding data sources, level of detail and evaluation is not given.

Consequently, no method is available for the fast creation of freely scalable vehicle architectures with multiple component variants and drivetrain concepts. To enable the fast creation of vehicle architectures at the beginning of the development stage, geometric substitute models for the component sizes and distances between them are the basis. Therefore, this paper targets two questions. Firstly: How are geometric substitute models developed and evaluated for components and distances of combustion, hybrid and electric drivetrain concepts? Secondly: What are the resulting model accuracies, using the developed methods? These geometric substitute models mark the foundation for initial architecture investigations.

\section{Method for the development of geometric substitute models}

In an abstract way, substitute models transfer an input into an output. The geometric substitute models convert one or more input parameters or requirements into component sizes or distances between them.

With the focus on the main dimensions, the geometric substitute models describe and approximate the complex geometries with cuboid, cylindrical and other simplified shapes.

For the creation of new substitute model, the authors developed the following process (Figure 1). This divides into the definition of input parameters, the determination of dimensions and subdimensions, the modelling of coherences and the evaluation of the created models. 


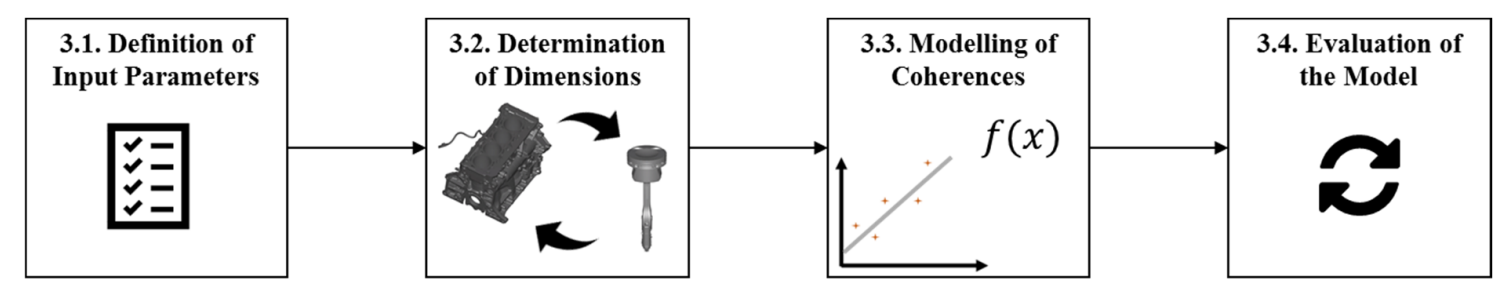

Figure 1. Procedure for the development of geometric substitute models

\subsection{Definition of input parameters}

Customer, concept and component requirements are available as input parameters for the development of geometric substitute models (Figure 2). The main difference between the input parameters is the effort involved in their determination and the level of detail. On one hand, the effort for the definition of customer requirements is relatively low. The use of customer requirements as a model input is legitimate, as the concept and component requirements correlate. For example, the capacity of the engine cooler depends on the engine power, which is dependent on the maximum speed. However, the indirect consideration leads to a limited accuracy. On the other hand, it is more difficult to determine the component requirements, as additional models and simulations might be necessary. However, these requirements offer a higher level of detail than customer requirements. The effort for determination and the level of detail of concept requirements ranges between these poles. Due to the focus at the early stage of Vehicle Architecture Design, the target is mainly on using customer and concept requirements for the development of the geometric substitute models. This phase usually lacks information about component requirements.

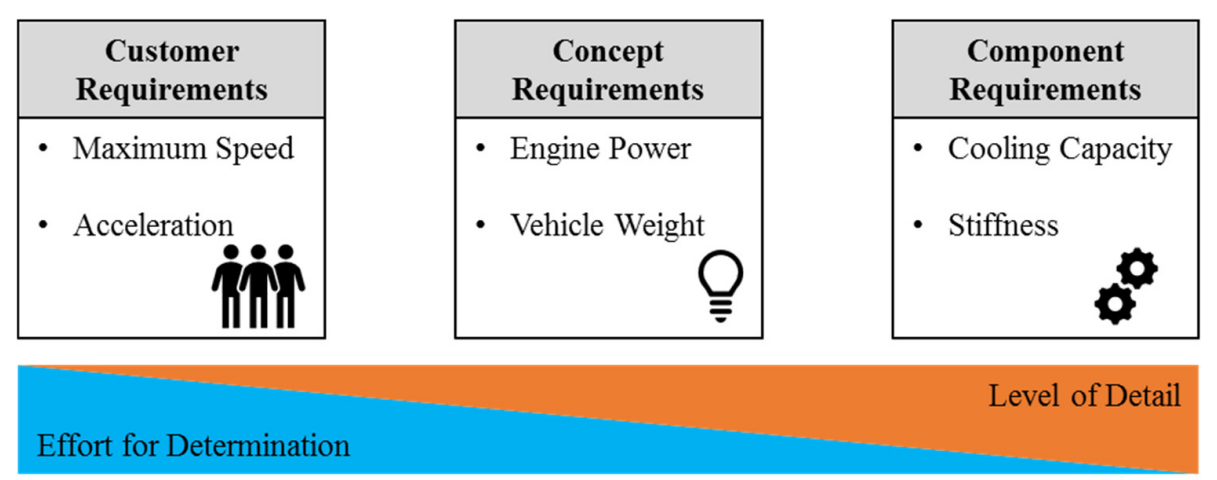

Figure 2. Different types of input parameters

\subsection{Determination of dimensions}

Before modelling coherences, it is important to determine the granularity of the dimensions.

One approach is to consider the overall dimensions of a component (no. 5, Figure 3). Therefore, the measuring effort is limited. However, the measuring accuracy is lower, as mounts and attachments lead to less standardized component shapes. Additionally, the measurement deviations are higher for larger dimensions.

Another approach is to divide the component into subcomponents. These subdimensions add up to the overall component dimensions using dimensional chains. As displayed in Figure 3, the bore diameter, the cylinder spacing and the overhang add up to the engine length. Besides the overhang, the subdimensions are more standardized. Therefore, dismantling the components increases the measuring accuracy. On the other hand, it increases the number of measurements.

The definition of the granularity is not only based on the advantages and disadvantages but also on the component shapes and the available data.

For distances between components, a division into subdimensions is not useful. 


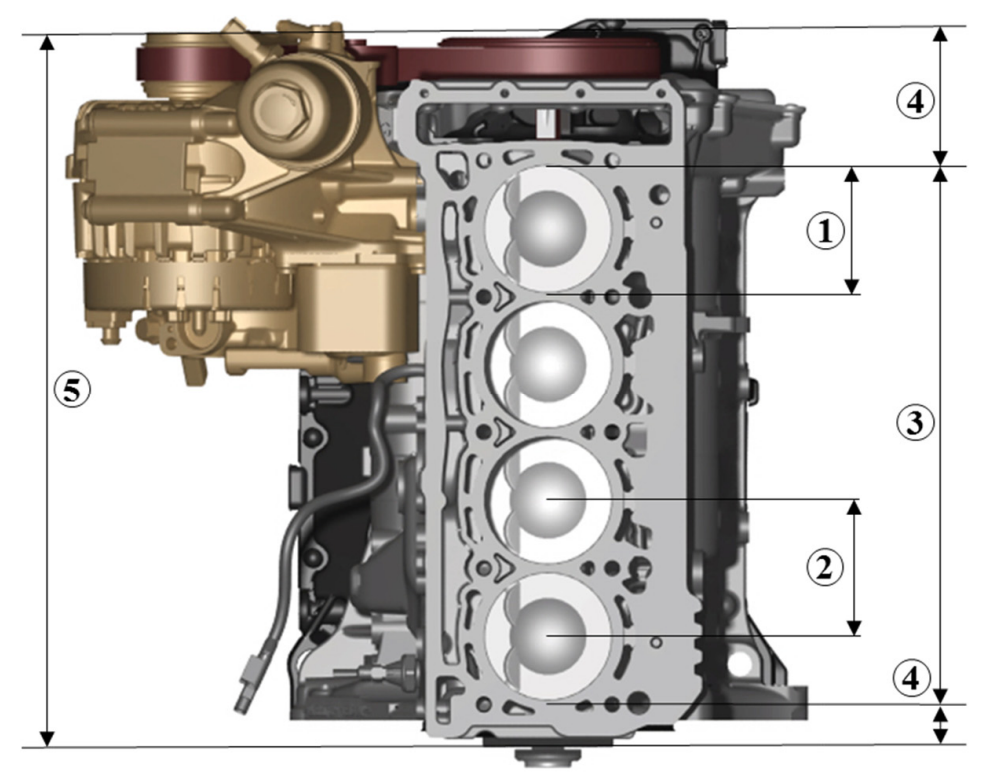

(1) Bore Diameter

(2) Cylinder Spacing

(3) Cylinder Block Length

(4) Overhang

(5) Engine Length

Figure 3. Division of dimensions into subdimensions

\subsection{Modelling of coherences}

The geometric substitute models define the coherences between the input parameters and the dimensions. Therefore, empirical (black-box) and physical (white-box) as well as semi-physical (greybox) approaches are possible (Schorn, 2006).

\subsubsection{Empirical modelling}

Empirical models define the coherences between input data and output data, using statistical methods. Based on a multitude of data sets, the statistic model describes the most likely coherence between the input parameters and the dimensions. For geometric substitute models, the empirical coherences can either be regression equations or constant values derived from a normal distribution (Figure 4).

For both, the first step is the measurement of the dimensions from existing vehicles. For each of the dimensions, it is necessary to identify and store the relevant input parameters.

With the measured dimensions and the documented requirements, the initial target is to generate a multiple linear regression model. Within a developed MATLAB tool, the dependent variable defines the dimension to be modelled. Using the backwards elimination method, the user selects a multitude of explanatory variables from the predefined input parameters. The explanatory variables can be metric (e.g. engine power) or binary (e.g. fuel type). Prior to the creation of the regression, the Variance Inflation Factor (VIF) indicates mutual influences of explanatory variables. As a result, explanatory variables with a VIF higher than 10 are successively eliminated (Wooldridge, 2013). Using the reduced set of explanatory variants, the tool outputs the distribution of the residuals. Only normal distributed residuals fulfil the requirement of homoscedasticity, which is elementary for a linear regression (Fahrmeir et al., 2013). Afterwards, the tool creates the equation for the linear regression. Besides constant and gradient terms for all explanatory variables, it considers interaction terms within the variables. Based on this equation, the least squares method derives the regression function. Accordingly, Cook's distance method identifies and removes outliners. The bisquare-weights method reduces further effects of outliners. The F-test and its P-value evaluate the overall statistical significance of the regression. Therefore, a P-value of $5 \%$ is set as a limit (Stoetzer, 2017). The F-test, the T-test and their $\mathrm{P}$-values analyse the statistical significance of each explanatory variable. The tool iterates the regression, after the elimination of insignificant variables. Subsequently, the adjusted coefficient of determination $\left(\operatorname{adj}-R^{2}\right)$ indicates the coverage of the real data by the regression. The normalized mean absolute error (nMAE) applies for the analysis of the model accuracy. In addition, a low difference between the nMAE and the root mean squared error (RMSE) indicates a uniform distribution without outliners (Chai and 
Draxler, 2014). The requirement of homoscedasticity makes the consideration of relative errors obsolete. Finally, the tool outputs an equation for the coherence between the dependent variable and the explanatory variables.

In the event that the regression function shows no significance, it is possible to derive a constant value. This means, that a dimension of a component or subcomponent and its variants is independent of the input parameters. Focussing solely on the automotive industry, there is only a limited scattering of the dimensions. Therefore, the MATLAB tool generates a normal distribution for the contemplated dimension. The limit of 1.96 times the standard deviation removes outliners. Thereafter, the variance as well as the difference between the mean and the median give an evaluation of the normal distribution. The Kolmogorov-Smirnov Test is another method for the interpretation and evaluation of the results. As a result, the nMAE outputs the model accuracy.

Most commonly, the regression-based models indicate significance for overall dimensions, such as the engine length. The significance is equally high for the dimensions of active subcomponents, such as for instance the length of the cylinder block. For passive (sub-) dimensions, like the thickness of the engine belt drive, constant values show good results.

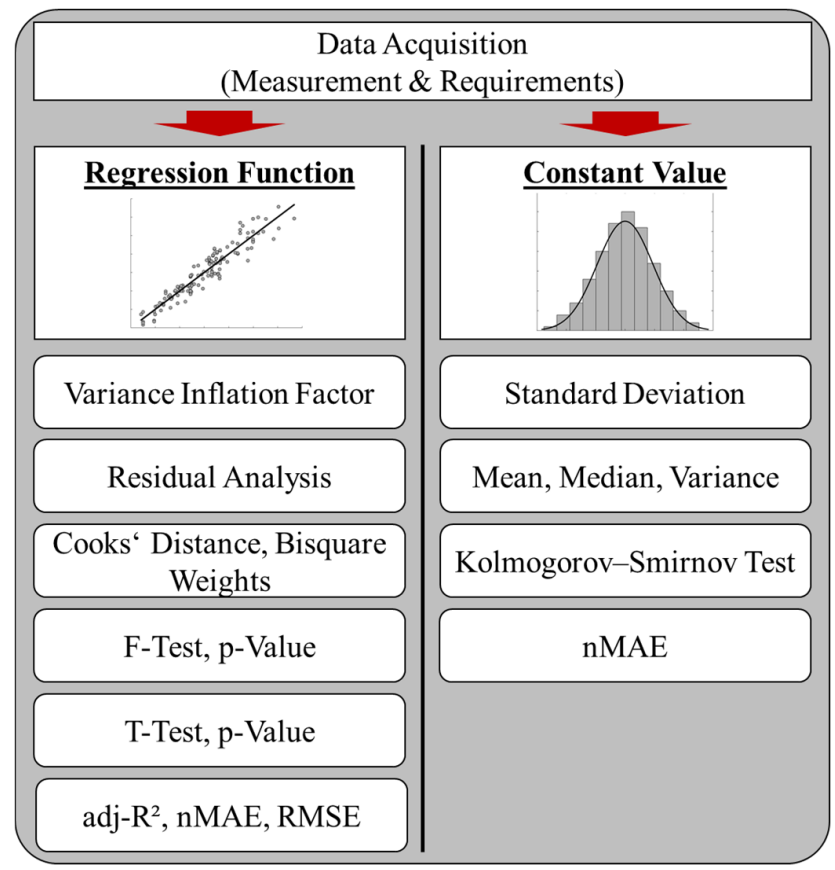

Figure 4. Procedures for the empirical modelling of geometric substitute models

\subsubsection{Physical modelling}

Physical models link the input data and output data using physical coherences. Therefore, a dismantling of the component into subcomponents and their dimensions is inevitable. For all dimensions, a physical equation defines the physical principals among the input data and the output data. For example, a physical equation describes the coherence between engine torque and gear wheel and shaft diameters of a gearbox. As a result, the equations for the subdimensions combine to a model for the overall dimension.

\subsubsection{Semi-physical modelling}

The linkage of empirical and physical modelling constitutes semi-physical modelling. Like physical models, the dismantling of components into subcomponents is inevitable. Subsequently, the physical approach models some of the dimensions. This usually relates to the active subcomponents and their dimensions. However, empirical regressions could also model active subcomponents. The determination of model parameters and passive dimensions is based on empirical constant values. 


\subsubsection{Comparison of the modelling types}

For geometric substitute models, three different modelling types are available. However, their application is dependent upon the amount of data and the possibility of dividing components into subcomponents. In addition, the complexity of the physical principles and the available input parameters affect the selection of the method. Another criteria is the computation time.

Empirical modelling requires a large amount of data (Wuppermann, 2016). However, this method is suitable for components with complex physical coherences, such as combustion engines. The application of the method is possible for overall dimensions as well as for subdimensions. Besides component requirements, the method allows the use of concept and customer requirements as inputs. The use of customer and concept requirements is advantageous as these are easier to determine, especially for competitor vehicles. However, the use of existing data limits the empirical models to a minimum of extrapolation. Due to the black-box modelling, the computation time is low.

Physical models apply to components with limited data availability, but also limited complexity of the physical coherences. Furthermore, the dismantling of the components and dimensions into subcomponents and subdimensions is inevitable. Input parameters are solely component requirements. Therefore, the determination of the input parameters requires other models and simulations beforehand. Compared to empiric models, interpolation is not a limitation. However, the computing time is higher. Physical, geometric substitute models are often unfeasible as it is impossible to model all parameters and dimensions solely physically.

In that case, semi-physical modelling is used. Therefore, the advantages and disadvantages of the empirical and physical models apply to the respective subdimension.

\subsection{Evaluation of the model}

The evaluation of the created geometric substitute models differs, depending on the modelling type. The nMAE analyses the accuracy of empirical modelled dimensions and subdimensions, using all available data. To assess the general validity of the models, an Out-of-Sample-Test (OOST) is executed (Tashman, 2000). Within the evaluation, the regression or constant value is based on $75 \%$ to $90 \%$ of the available data. Consequently, the remaining evaluation data serves as an input for the created regression equation. Subsequently, the evaluation of general validity consists of the comparison of the resulting dimensions with the real dimensions of the evaluation data. For constant values, the evaluation compares the derived value with the values of the evaluation data. To avoid the influence of random data selection, the OOST iterates multiple times and outputs the average deviation. This cross-validation serves for the assessment of the models' general validity. However, the OOST is infeasible for the compound overall dimensions and is therefore only conducted for all individual or subdimensions. Hence, the implemented model considers all available data.

The evaluation of physical models always uses all available data. Based on the requirements of the available data, the physical function calculates the dimensions. Afterwards, the nMAE of the modelled dimensions and the real dimensions defines the modelling accuracy of physical models.

The semi-physical models use both approaches for evaluation, depending on the modelling type of a dimension.

For dismantled dimensions, the evaluation methods test all subdimensions and the compound overall dimensions. This evaluates the overall performance and the mutual influences of inaccuracies.

\section{Resulting geometric substitute models}

Based on the presented method, the authors developed geometric substitute models for over 25 components and component variants as well as distances between components in the vehicle's front section. Figure 5 gives an extract of modelled components and distances. With a focus on the early stage of Vehicle Architecture Design, the authors mainly used customer and concept requirements as inputs. For the data, the automotive benchmarking database A2Mac1 is the main source. For the years 2010 to 2017, the database contains over 320 vehicle disassembles of over 45 automotive manufactures (A2MAC1 EURL, 2018). Using the example of combustion engines and electric machines, the following gives an insight into the developed models. 


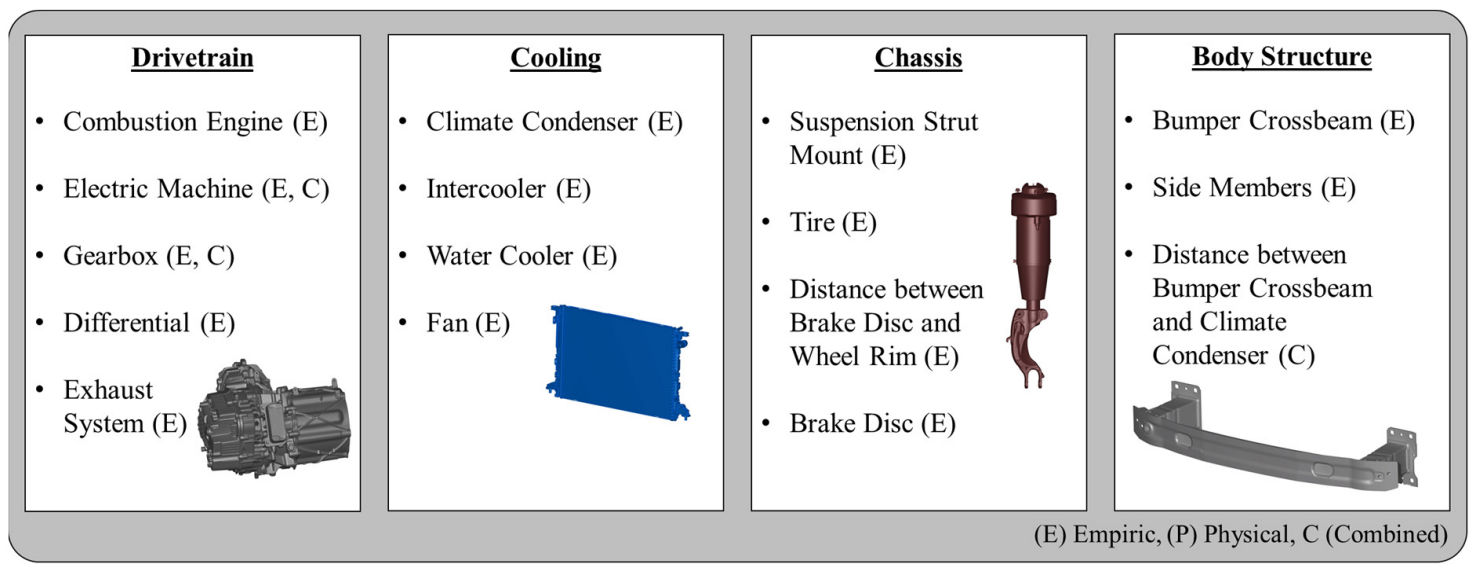

Figure 5. Extract of the created geometric substitute models

\subsection{Combustion engines}

The target of the substitute models for combustion engines is the output of the engine length, width and height.

To increase the modelling accuracy, the engine is divided into subcomponents (Figure 3). The A2Mac1 database provides measurements and input parameters for over 300 combustion engines, from vehicles from 2010 to 2017.

The main input parameters are the engine torque and the engine power. As vehicle manufacturers offer the same engine with different performances, it is important to identify the peak values. The database is split mainly into in-line and V-type engines, depending on the subdimension. The fuel type (petrol/diesel) and the charging (naturally aspirated/supercharged) are either considered as input parameters (binary variable) or by segmentation of the database.

For 227 of the over 300 engines, the authors measured over 20 subdimensions and gathered over 10 types of input parameters. Afterwards, the authors created an empirical model for the engine. The following exemplifies the modelling in a longitudinal direction for in-line engines. For the engine width and height as well as for V-type engines, similar results exist.

Figure 6 shows a regression between the engine torque and the engine displacement. In this respect, the database is divided into naturally-aspirated petrol, supercharged petrol and supercharged diesel engines. The engines within each of these groups have similar torque and power characteristics. Therefore, the elimination of the engine power as an input parameter avoids a mutual influence with the engine torque. The results indicate that naturally aspirated petrol engines have the highest engine displacement for the same torque requirement.

Subsequently, a normal distribution reveals the average cylinder swept volume (Figure 6). The different design philosophies of the vehicle and engine manufacturers induce the variation in the cylinder swept volume (van Basshuysen and Schäfer, 2015). However, the resulting mean of $449 \mathrm{~cm}^{3}$ is close to $500 \mathrm{~cm}^{3}$, which is ideal for modular engine systems of 2 litres ( 4 cylinders), 3 litres (6 cylinders) and more (Huß, 2012). With this regression function and constant value, it is possible to calculate the number of cylinders based on a required engine torque and a definition of the engine, fuel and charging type. A constant value for the stroke-bore ratio allocates the cylinder swept volume by the bore diameter and the stroke (Figure 7). Previous studies also came to the outcome that in-line engines are long-stroke engines (Huß, 2012). The resulting bore diameter is an input for a regression function of the cylinder spacing. A similar coherence is described in literature (Köhler and Flierl, 2012). The number of cylinders, the bore diameter as well as the cylinder spacing gives a calculation for the cylinder block length. Adding a constant value for the overhang, the engine length is output. This is feasible, as the components of the overhang, such as the belt drive, depend less on the engine torque. The nMAE of the modelled length is $8.4 \%$ for R- and V-type engines. The models for the engine width and height show similar results. 

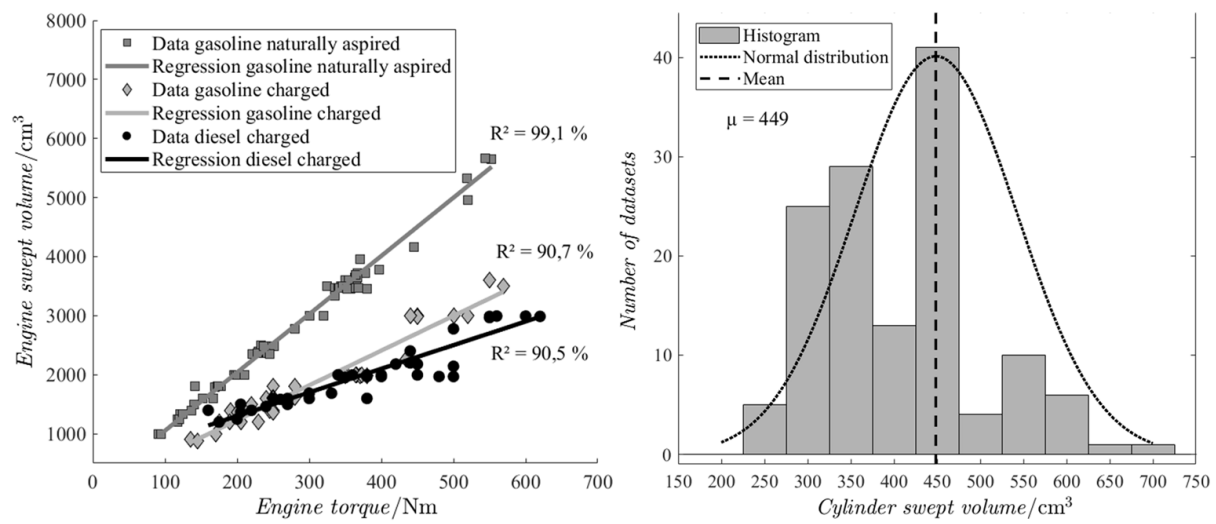

Figure 6. Regression between the engine swept volume and the engine torque (left) and normal distribution for the cylinder swept volume (right)
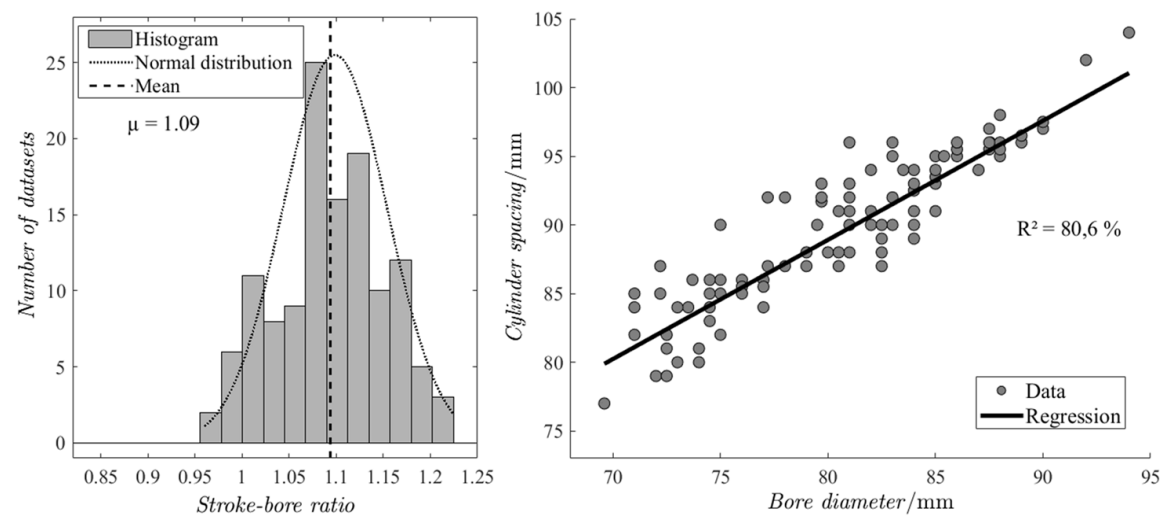

Figure 7. Normal distribution for the stroke-bore ratio (left) and regression between the cylinder spacing and the bore-diameter (right)

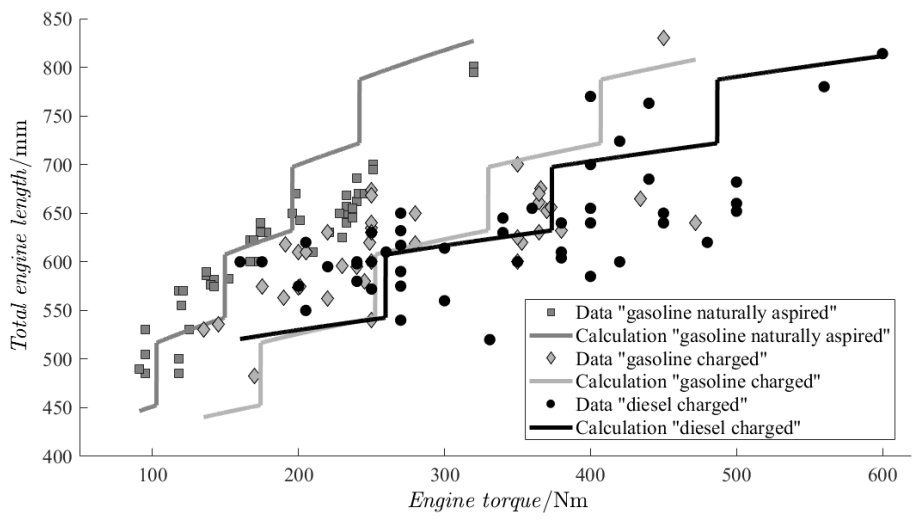

Figure 8. Modelled engine length in dependency of the engine torque

\subsection{Electric machines}

For the electric machines, the authors modelled an empirical geometric substitute model and enhanced a semi-physical one.

The A2Mac1 database and datasheets from suppliers provide in total 43 electric machines for the empirical model. The application field of all electric machines considered is within the automotive industry. Asynchronous (ASM) and permanent synchronous (PSM) machine types are in some cases 
distinguished as a binary variable. The developed model is based on the overall dimensions, instead of subdimensions.

Figure 9 shows the regression function for the overall volume, including the housing (without stiffeners), of the electric machine. The volume depends on the nominal machine torque and the nominal machine power as metric variables. The input voltage of the considered electric machines ranges from $115 \mathrm{~V}$ to $700 \mathrm{~V}$. Instead of using the input voltage as an explanatory variable, the power of each engine is normalized to $400 \mathrm{~V}$. This is the most common input voltage within the available engines. In contrary to combustion engines, the machine torque and the machine power parameters do not have a strong mutual influence. The machine type is a binary variable within the model. Like in literature, the torque shows a greater impact on the resulting volume than the power, for both machine types (Müller and Ponick, 2012). As a result, asynchronous machines require more volume than permanent synchronous machines.

A regression function for the length-diameter ratio allocates the volume to the machine length and diameter. This function depends on the maximum rotational speed. In this respect, the database consists of only 24 electric machines, as the remaining 19 do not provide information about the maximum speed. The result shows that high-speed electric machines are low in diameter, to avoid high circumferential forces. The nMAE is $12.5 \%$ for the machine length and $7.2 \%$ for the diameter.
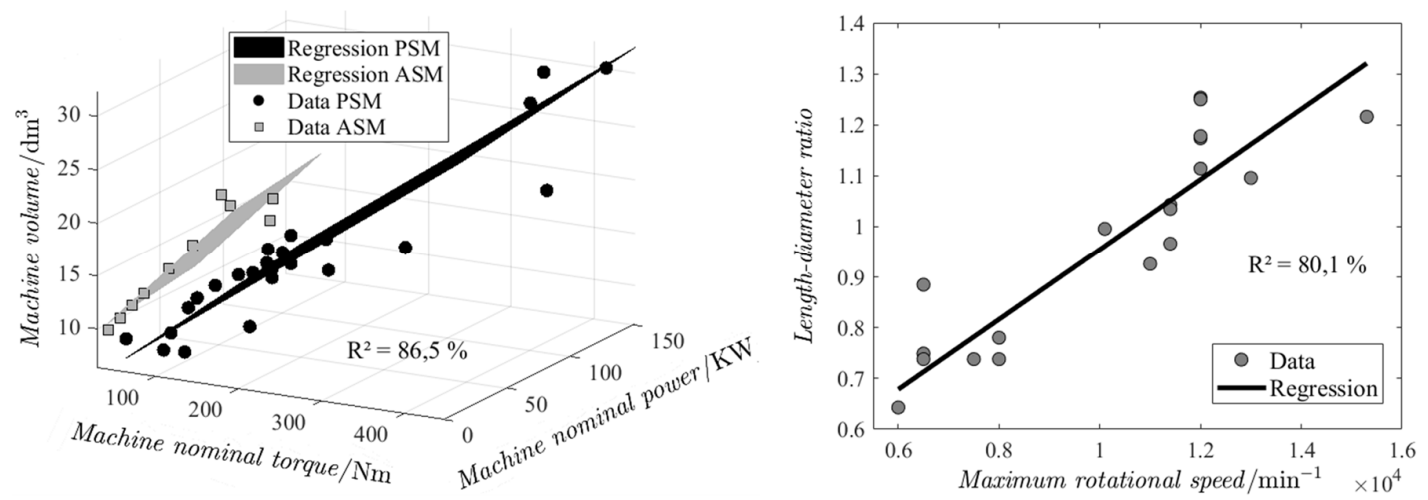

Figure 9. Regression between the machine volume and the machine nominal torque and power (left) and regression between the length-diameter ratio and the maximum rotational speed (right)

The physical equations for the rotor and stator dimensions enable the semi-physical approach for the electric machines. The model of Horlbeck (2018) already calculates the rotor as well as stator length and diameter, as part of an efficiency map simulation. This model itself is a semi-physical approach, as some physical equations require constant values. Using the regression function for the length-diameter ratio and constant values for the winding head and casing, it was possible to enhance the existing model. Consequently, the model outputs the overall length and diameter. For the machine length and the diameter, the results of the semi-physical model show a nMAE of $11.6 \%$ and $19.9 \%$.

\subsection{Influences on the model accuracy}

The presented models and the evaluation show high accuracies. Nevertheless, several influences on the accuracy of geometric substitute models exist.

The amount of available data sets limits the accuracy of the empirical substitute models. For valid results, the target is to use at least 30 to 40 datasets. However, especially for BEV components, existing data is not extensive. Besides the amount of data, the measurement accuracy affects the results. Even though the A2Mac1 database offers high-quality benchmark analysis, the measures can be imprecise for complex and unstandardized components, such as the exhaust system. To increase the accuracy, dubious datasets are not included in the database.

Modular strategies of the automotive manufacturers further affect the results. The use of a component for multiple requirements increases the production quantity. Therefore, the challenge is the identification of the maximal requirement to the component as input. 
Compared to empirical models, semi-physical models often show slightly lower accuracy. Empirical models include the design nuances of the automotive industry. One advantage is that semi-physical models require less data for the modelling.

\subsection{Discussion of geometric substitute models}

The results of the combustion engines and the electric machines show the ability of empirical and semiphysical geometric substitute models to determine dimensions. The accuracy of the developed geometric substitute models ranges from $80 \%$ to $97 \%$. The lower accuracies occur mainly within the cooling system due to the high design flexibility of the plastic side parts. Nevertheless, the accuracies are high enough for use at the early stage of the vehicle concept development.

The empirical and semi-physical models output the statistically most likely dimension for an inputted requirement. Consequently, the geometric substitute models do not output the best available solution on the market. In addition, the models do not directly include the ability of control units and software to slightly increase component capabilities without changing the geometry. As a result, the geometric substitute models are a robust and conservative solution.

The geometric substitute models define the coherences between a limited number of input parameters and the dimensions. This enables use within the early phase of the vehicle concept development. In contrary, the models lack the consideration of all requirements, as well as design and manufacturers philosophies.

Consequently, the models apply only for the initial phase of the architecture design, as later on, more requirements and detailed geometries need to be considered.

\section{Conclusion and outlook}

Due to the high number of requirements and component variants, the determination of component dimensions as well as distances between them is difficult and time-consuming at the beginning of vehicle concept development. Therefore, the authors developed geometric substitute models based on the presented method. In doing so, they considered components and distances of the vehicle front from combustion, hybrid and electric drivetrains.

At the initial stage of Vehicle Architecture Design, concept engineers can use these models to scale the component dimensions and distances between components. These dimensions serve as starting point for architecture investigations. Due to numerous substitute models, a comparison between different components and component variants is possible. In addition, concept engineers analyse the effects of changes within the requirements on the dimensions. As a result, the use of geometric substitute models reduces the development time and capacities. At $80 \%$ to $97 \%$, the model accuracies are high enough for the early design phase. As the development progresses, simulations and designed components will replace the dimensions taken from the substitute models.

As an outlook, the authors will use the created substitute models for the automated and scalable design of vehicle architectures with multiple drivetrain concepts. At the beginning of the development, this tool supports concept engineers with architecture investigations. Through the evaluation of the tool, the authors will also assess substitute models in their applicability.

\section{Contributions}

The lead author Matthias Felgenhauer developed the idea for geometric substitute models. Based on that idea, he created the method for the generation of the models, including the statistic procedure and the evaluation. Raul Marksteiner and Florian Schneider supported in the development of the statistic procedure. During their Masters' theses, both generated geometric substitute models by statistic data evaluation, with close discussion of the procedure, the concept and the results with their thesis advisor Matthias Felgenhauer. Christian Angerer supported in the comparison of empirical and semi-physical models of electric machines. Markus Lienkamp contributed to the method, the proofreading and strongly agrees with the concept. 


\section{Acknowledgement}

This article contains results of the Master's thesis of Ömer Tan. Furthermore, it includes conceptual design from Frank Züge (Head of Department) and Frank Schöpe (Team Leader) from the Department for Front and Rear Vehicle Concepts MLB at the AUDI AG. In addition, the authors would like to thank Wilhelm König from A2Mac1 EURL for the support and access to the A2Mac1 automotive benchmarking database. AUDI AG and Technical University of Munich have sponsored the project.

\section{References}

A2MAC1 EURL (2018), A2macl. Automotive Benchmarking. [online] A2Mac1. Available at: https://www.a2mac1.com/ (accessed 25.02.2018).

Chai, T. and Draxler, R.R. (2014), "Root mean square error (RMSE) or mean absolute error (MAE)? Arguments against avoiding RMSE in the literature", Geoscientific Model Development, Vol. 7 No. 3, pp. 1247-1250. https://doi.org/10.5194/gmd-7-1247-2014

Fahrmeir, L., Kneib, T., Lang, S. and Marx, B. (2013), Regression: Models, Methods and Applications, Springer Berlin Heidelberg, Berlin, Heidelberg.

Felgenhauer, M., Stocker, J. and Lienkamp, M. (2017), "New approach for architecture design of high variable vehicle portfolios", Proceedings of the ASME 2017 International Mechanical Engineering Congress and Exposition, Tampa, USA, November 3 - 9. https://doi.org/10.1115/IMECE2017-71155

Fuchs, J. (2014), Analyse der Wechselwirkungen und Entwicklungspotentiale in der Auslegung elektrifizierter Fahrzeugkonzepte, PhD thesis, Institut für Maschinen- und Fahrzeugtechnik, Lehrstuhl für Fahrzeugtechnik, Technische Universität München, München.

Horlbeck, L. (2018), Auslegung elektrischer Maschinen für Automobile Antriebsstränge unter Berücksichtigung des Überlastpotentials, $\mathrm{PhD}$ thesis, Institut für Maschinen- und Fahrzeugtechnik, Lehrstuhl für Fahrzeugtechnik, Technische Universität München (unpublished).

Huß, M. (2012), Übertragung von Motoreigenschaften mit Hilfe charakteristischer Skalierfunktionen zur Simulation verschiedener Varianten von Ottomotoren, $\mathrm{PhD}$ thesis, Institut für Energietechnik, Lehrstuhl für Verbrennungskraftmaschinen, Technischen Universität München.

Köhler, E. and Flierl, R. (2012), Verbrennungsmotoren: Motormechanik, Berechnung und Auslegung des Hubkolbenmotors, ATZ/MTZ-Fachbuch, 6th ed., Vieweg+Teubner Verlag / Springer Fachmedien Wiesbaden GmbH Wiesbaden, Wiesbaden. https://doi.org/10.1007/978-3-8348-8309-4

Kuchenbuch, K. (2012), Methodik zur Identifikation und zum Entwurf packageoptimierter Elektrofahrzeuge, PhD thesis, Technischen Universität Carolo-Wilhelmina zu Braunschweig.

Matz, S. (2015), Nutzerorientierte Fahrzeugkonzeptoptimierung in einer multimodalen Verkehrsumgebung, PhD thesis, Institut für Maschinen- und Fahrzeugtechnik, Lehrstuhl für Fahrzeugtechnik, Technische Universität München.

Müller, G. and Ponick, B. (2012), Grundlagen elektrischer Maschinen, Elektrische Maschine, John Wiley \& Sons, Hoboken.

Ried, M. (2014), Lösungsraumanalyse für Plug-In-Hybridfahrzeuge hinsichtlich Wirtschaftlichkeit und Bauraumkonzept, $\mathrm{PhD}$ thesis, Universität Duisburg-Essen, Duisburg-Essen.

Schorn, M. (2006), "Modelle zur Beschreibung des Fahrzeugverhaltens", In Isermann, R. (ed.), FahrdynamikRegelung: Modellbildung, Fahrerassistenzsysteme, Mechatronik; mit 28 Tabellen, Kraftfahrzeugtechnik, Vieweg, Wiesbaden, pp. 27-46. https://doi.org/10.1007/978-3-8348-9049-8_2

Stoetzer, M.-W. (2017), Regressionsanalyse in der empirischen Wirtschafts- und̄ Sozialforschung, Springer Berlin Heidelberg, Berlin, Heidelberg. https://doi.org/10.1007/978-3-662-53824-1

Tashman, L.J. (2000), "Out-of-sample tests of forecasting accuracy: An analysis and review", International Journal of Forecasting, pp. 437-450. https://doi.org/10.1016/S0169-2070(00)00065-0

van Basshuysen, R. and Schäfer, F. (2015), Handbuch Verbrennungsmotor: Grundlagen, Komponenten, Systeme, Perspektiven, ATZ/MTZ-Fachbuch, 7th ed., Springer Vieweg, Wiesbaden. https://doi.org/10.1007/978-3-65804678-1

Wooldridge, J.M. (2013), Introductory econometrics: A modern approach, 5th ed., South-Western Cengage Learning, Mason Ohio.

Wuppermann, A. (2016), Empirische Ökonomie I. [online] Wuppemann, A. Available at: http://www.micmetr.econ.uni-muenchen.de/lehre1/wintersemester-2017_18/emp1/index.html

Matthias Frank Felgenhauer, Research Associate

Technical University of Munich, Institute of Automotive Technology

Jahnstr. 23, 85521 Ottobrunn, Germany

Email: felgenhauer@ftm.mw.tum.de 